\section{Governo local e serviços odontológicos: análise da desigualdade na utilização}

\author{
Local government and public dental health \\ services: an analysis of inequality in use
}

\author{
Gobierno local y servicios odontológicos: análisis \\ de la desigualdad en el uso
}

\author{
${ }^{1}$ Instituto de Saúde Coletiva, \\ Universidade Federal da \\ Bahia, Salvador, Brasil. \\ 2 Faculdade de Odontologia \\ Universidade Federal da \\ Bahia, Salvador, Brasil. \\ Correspondência \\ F. F. Soares \\ Instituto de Saúde Coletiva \\ Universidade Federal da \\ Bahia. \\ Rua Theodomiro Baptista \\ 126 , apto. 501 \\ Salvador, $B A$ \\ 41940-320, Brasil. \\ felipefagundes@outlook.com
}

\section{Abstract}

The aim of this study was to identify factors associated with the use of primary and specialized public dental health services and private services. A population-based household survey was conducted in two cities of Bahia State, Brazil. Key informants provided data on socioeconomic variables and use of dental health services. Organization of the local public dental health service was ranked as worse versus better. Univariate and multivariate polytomous logistic regression was performed. Of the total of 1,290 individuals, $38.76 \%$ used private services, $33.80 \%$ used public primary care, and $17.29 \%$ used both primary care and the Center for Dental Specialties. Less use of both primary care and specialized public services was associated with lower education $(O R=$ 1.47; 95\%CI: 1.03-2.10) and worse organization of services (OR = 1.74; 95\%CI: 1.22-2.48), when compared to the exclusive use of primary care. The study showed inequality in the use of dental services, even when comparing more homogeneous groups, namely users of public services.

Health Inequalities; Secondary Care; Health Services Accessibility; Dental Care; Local Government
Felipe Fagundes Soares 1

Sônia Cristina Lima Chaves 1,2 Maria Cristina Teixeira Cangussu ${ }^{2}$

\section{Resumo}

O objetivo deste estudo foi identificar fatores associados à utilização dos serviços odontológicos, públicos (básicos e especializados) e privados. Foi realizado inquérito populacional de base domiciliar em dois municípios da Bahia, Brasil. Informanteschave forneceram dados socioeconômicos e de utilização dos serviços odontológicos (desfecho). A organização do serviço público odontológico local foi classificada em pior/melhor. Realizou-se regressão logística politômica uni e múltipla. Do total de 1.290 indivíduos, 38,76\% usaram o serviço privado, 33,80\% atenção básica e 17,29\% atenção básica e o Centro de Especialidades Odontológicas (CEO). Um perfil de vulnerabilidade social foi associado ao uso do serviço público, quando comparado ao privado. Menor escolaridade $(O R=1,47$ IC95\%: 1,03-2,10) e pior organização do serviço $(O R=1,74 ;$ IC95\%: 1,22-2,48) foram associados ao menor uso da rede de serviços atenção básica e CEO em comparação ao uso exclusivo da atenção básica. A desigualdade na utilização dos serviços odontológicos foi observada mesmo quando comparados grupos mais homogêneos, como os usuários dos serviços públicos.

Desigualdades em Saúde; Atenção Secundária à Saúde; Acesso aos Serviços de Saúde; Assistência Odontológica; Governo Local 


\section{Introdução}

A desigualdade no acesso e utilização de serviços odontológicos tem sido evidenciada em estudos nacionais 1,2,3,4 e internacionais 5 . Ela pode ser produzida pela posição social ocupada pelos indivíduos como resultado de lutas e relações estabelecidas com outros agentes 6 . Existem evidências de que pessoas com maior vulnerabilidade social são mais acometidas pelas morbidades em saúde bucal e têm maior acúmulo de necessidades ${ }^{2}$. Assim, indivíduos com maior capital econômico, cultural e social usam melhor os serviços de saúde 6 , inclusive os odontológicos 7 , principalmente quando se trata daqueles de acesso mais difícil, como a atenção especializada.

A atenção especializada está proposta pela Política Nacional de Saúde Bucal (PNSB) através dos Centros de Especialidades Odontológicas (CEO), unidades de referência e contra-referência da atenção básica para diagnóstico e procedimentos mais complexos 8. Entre 2004 e 2012 foram implantados 943 CEO em 771 municípios brasileiros ${ }^{9}$. O uso desta rede de serviços tem conferido ao usuário maior integralidade do cuidado, embora esta seja comprometida pela baixa oferta e dificuldade de acesso a este serviço 10 .

Ser atendido previamente na Estratégia Saúde da Família (ESF), encaminhado com ficha de referência, realizar tratamento endodôntico, ter até 35 anos e morar numa cidade com cobertura da ESF maior que $50 \%$ foram apontados como fatores que favorecem o uso dos serviços de saúde bucal com maior integralidade 11 .

A literatura acerca da atenção especializada em saúde bucal e os determinantes do seu uso ainda é incipiente, entretanto supõe-se que eles estejam associados a elementos da organização do serviço e às características relativas ao usuário 12 .

Existem evidências teóricas 12 e empíricas $10,11,13,14,15$ de que o modo como a política nacional é implementada, organizada e gerida no nível local é importante para a melhor utilização dos serviços pela população que mais os demandam. Entretanto, a literatura ainda carece de estudos analíticos que confirmem esta associação.

As pesquisas sobre desigualdade no uso dos serviços odontológicos são muito diversas quanto aos seus determinantes. Estes podem variar desde o local em que a pesquisa foi feita (país e zona rural/urbana) até a natureza do serviço avaliado (público ou privado, atenção básica ou atenção especializada, médico ou odontológico) ${ }^{12}$. Autores que investigaram a utilização sem especificar a sua natureza, encontraram que a cor da pele preta é um fator limitante entre os idosos 16 e que a maior renda e escolaridade estão associados positivamente, independente da faixa etária 17. Estas características são semelhantes às encontradas entre os usuários da assistência odontológica privada, a mais acessada no país 2,18 .

Por outro lado, o perfil dos usuários das Equipes de Saúde Bucal (ESB) da ESF é composto por mulheres, idosos, desempregados, pobres, negros, não cobertos por planos de saúde e com a autopercepção da saúde ruim. Portanto, teoricamente, a ESF tende a promover um uso mais equitativo 1,2,3,19.

No Brasil, o atendimento odontológico no Sistema Único de Saúde (SUS) é apontado como uma intervenção da PNSB, que pode ter efeito favorável na diminuição das desigualdades em saúde 20. Esta redução, registrada com base nos dados da Pesquisa Nacional por Amostra de Domicílios (PNAD) de 1998 e 2008, foi acompanhada pelo aumento no uso dos serviços 1, em virtude da maior oferta assistencial, da melhoria das condições de vida da população 21 , da diminuição do desemprego e do aumento da renda média familiar 1 .

Nesse contexto, ressalta-se que a desigualdade na utilização dos serviços odontológicos compromete a integralidade do cuidado, dificultando que as pessoas tenham suas necessidades de saúde satisfeitas. Assim, tendo em vista a importância do enfrentamento destas desigualdades, foi objetivo desse estudo identificar fatores associados à desigualdade na utilização dos serviços odontológicos, públicos (básicos e especializados) e privados, em municípios com cobertura da ESF estimada em $100 \%$.

\section{Metodologia}

Realizou-se um inquérito domiciliar de base populacional em dois municípios médio porte (50.000 a 100.000 habitantes), localizados na região metropolitana de Salvador, Bahia, Brasil, com cobertura potencial estimada em $100 \%$ da ESF e que implantaram o CEO há pelo menos quatro anos. Dois municípios foram indicados pela Secretaria Estadual de Saúde por serem bem avaliados de acordo com os relatórios de acompanhamento dos CEO's e de visitas técnicas da Coordenação Estadual de Saúde Bucal.

\section{Caracterização dos municípios}

O município A tem uma população de 42.153 habitantes, dos quais $13,8 \%$ são analfabetos e o rendimento domiciliar médio per capita é de R\$ 371,00. Existem 12 Unidades de Saúde da Família (USF) e um CEO tipo I. Os 18 dentistas que traba- 
lham no governo local estabelecem uma relação de 0,4 dentistas-SUS $/ 10^{3}$ habitantes. Já o município B possui 51.077 habitantes, com uma proporção de $11,1 \%$ de analfabetismo e renda média domiciliar per capita de R\$419,00. Há 15 USF e um CEO tipo I, nos quais trabalham 24 dentistas, ou seja, 0,5 dentistas-SUS $/ 10^{3}$ habitantes. Os municípios pesquisados têm indicadores sociais semelhantes, especialmente o índice de Gini $(\mathrm{A}=0,41 ; \mathrm{B}=0,42)$ e $\mathrm{o}$ índice de desenvolvimento humano $(\mathrm{A}=0,657 ; \mathrm{B}=0,677) 22$.

Apesar de a Coordenação Estadual de Saúde Bucal avaliar positivamente o funcionamento dos CEO's em 2011, no município B a avaliação realizada por Rossi 14 encontrou baixo êxito na implementação deste serviço, com falta de planejamento técnico e apoio político. A análise do Triângulo de Governo de Carlos Matus 23, elaborada por esta autora 14 , revelou que a coordenação de saúde bucal trabalhava conforme a lógica de inserção no setor privado e tinha baixa capacidade de governo e governabilidade. Além disso, não havia uma central de marcação de consultas e o propósito de governo não previa o crescimento das ações públicas em odontologia. $\mathrm{O}$ CEO teve seu funcionamento ameaçado desde 2009, com cortes no financiamento, diminuição da produtividade e distanciamento das metas propostas pelo Ministério da Saúde 14.

Por outro lado, no município A houve êxito na implementação da PNSB, com apoio político da gestão municipal e planejamento técnico, após pressão exercida pelo Conselho Municipal de Saúde e pelos resultados do levantamento epidemiológico. A Coordenação de Saúde Bucal tinha formação de sanitarista e experiência na gestão, o que lhe dava alta capacidade de governo e habilidades no controle do processo de trabalho. $\mathrm{O}$ serviço funcionava segundo as diretrizes do Ministério da Saúde, e havia uma central de marcação de consultas 14 .

\section{Cálculo amostral}

Realizou-se uma amostragem de conglomerado em dois estágios, sendo o primeiro a seleção dos setores censitários e o segundo, os domicílios, selecionados de forma sistemática devido à ausência de um cadastro atualizado que possibilitasse uma seleção aleatória. Em cada município foram sorteados dez setores censitários, sendo sete urbanos e três rurais, de modo a garantir a proporcionalidade entre as zonas. Neste cálculo, utilizou-se o Censo Demográfico de 200024.

Para cada setor censitário, calculou-se a amostra dos domicílios elegíveis, tendo por objetivo estimar a proporção de pessoas que utilizaram a assistência odontológica. Para isso, considerou-se uma prevalência de 50\% (desconhecida), no nível de confiança de $95 \%$ e erro amostral de $4 \%$. Por setor, foram selecionados 50 domicílios na zona urbana e 45 na rural (tamanho mínimo para todos os setores).

Para este estudo, foi critério de inclusão procurar algum serviço odontológico no último ano. Como exclusão, usar somente o CEO, sem fazer previamente tratamento na atenção básica. Neste caso, os usuários podem ter conseguido acesso ao serviço por se tratar de uma urgência, ou através da amizade com os profissionais das equipes de saúde bucal - formas de entrada não investigadas por este trabalho.

\section{Coleta dos dados}

Agentes Comunitários de Saúde (ACS), treinados em duas oficinas com pré-teste da ficha, coletaram informações de todos os moradores com idade maior ou igual a 15 anos, no período de junho a setembro de 2011. Em cada domicílio, um informante-chave respondeu pelos demais moradores. A ficha conteve questões sócio-demográficas (beneficiário de programa social, renda mensal familiar, sexo, idade, etnia, escolaridade, local de residência, número de bens, cômodos e pessoas residentes no domicílio, tipo de organização do serviço de saúde bucal), e de utilização de serviços de saúde bucal (tipo de serviço utilizado e procedimentos realizados), referente ao período máximo de 12 meses anteriores ao momento da entrevista.

\section{Plano de análise}

A utilização, ou uso destes serviços, foi o desfecho principal. Neste trabalho ela foi compreendida como sinônimo de acesso ou como resultado da interação entre o comportamento do indivíduo que precisa do cuidado, do profissional que o conduz dentro do sistema de saúde e das próprias características deste sistema 12 .

O desfecho foi categorizado em quatro categorias: (a) tentou acesso em algum local e não conseguiu, (b) usou somente atenção básica, (c) usou setor privado (convênio e particular) e (d) usou atenção básica e o CEO, sendo esta última categoria considerada como referência por representar aqueles que conseguiram transitar por toda rede de serviços e atingiram, possivelmente, a integralidade pretendida quando necessitaram. Aqueles que usaram concomitantemente o serviço público e privado foram classificados como usuários deste último. Neste caso, entendese por integralidade a utilização oportuna dos serviços nos distintos níveis de atenção, quando necessitados 25 . 
As variáveis potencialmente associadas ao desfecho foram dicotomizadas. Para as discretas (idade, escolaridade, número de bens e de pessoas/cômodo), utilizou-se a mediana como ponto de corte (até 35 anos $=0$, mais de 35 anos $=1$; até 7 anos de estudo $=1$, mais de 7 anos de estudo $=0$; até 3 bens $=1$, mais de 3 bens $=0$; até 0,6 pessoas $/$ cômodo $=0$, mais de 0,6 pessoas $/$ cômodo $=1$ ) . A renda familiar mensal foi categorizada em até um salário mínimo $(=1)$ e mais de um $(=0)$, tendo como parâmetro o valor de R $\$ 545,00$ em 2011, ou US\$ 311,00 (Banco Central do Brasil. http:// www4.bcb.gov.br/pec/taxas/port/ptaxnpesq. asp?id=txcotacao, acessado em 27/Out/2013). A cor da pele foi dicotomizada entre negros $(=1)$ e não negros $(=0)$; o local de residência entre zona urbana $(=1)$ e rural $(=0)$, e quanto ao trabalho exercido, entre remunerado $(=0)$ e não remunerado $(=1)$.

A variável organização do serviço foi categorizada em melhor $(=0)$ e pior $(=1)$, conforme a análise da implementação da PNSB nestes municípios realizada por Rossi 14 (os elementos que fundamentam estas categorias foram descritos previamente na caracterização dos municípios) à luz do Triângulo de Governo ${ }^{23}$, que permitiu avaliar se o funcionamento do serviço estava de acordo com as diretrizes propostas pela política, se existia planejamento e apoio político para o projeto de implementação do CEO e como se deu a condução do processo de trabalho pelos gestores. Estas evidências, detalhadas na caracterização dos municípios, viabilizaram a classificação dos indivíduos residentes no município A como expostos a um serviço mais organizado e próximo das diretrizes da PNSB que aqueles moradores da outra localidade pesquisada.

Realizou-se proporção absoluta e relativa para a estatística descritiva. O teste qui-quadrado de Pearson determinou a diferença da prevalência de cada categoria do desfecho por variável independente. Como a variável resposta tem quatro categorias nominais, utilizou-se a regressão logística politômica 26. Esta análise possibilitou associar as variáveis independentes ao desfecho, que teve a categoria de referência (indivíduos que conseguiram transitar pela rede pública de saúde bucal, atenção básica e CEO) comparada a: (i) Aqueles que não conseguiram transpor as barreiras de acesso = uso do serviço odontológico público $v s$. barreiras de acesso;

(ii) Aqueles que usaram serviços de saúde bucal somente na atenção básica = somente atenção básica $v s$. atenção básica e CEO;

(iii) Aqueles que usaram o serviço odontológico privado $=$ público $\nu s$. privado.

O modelo de regressão logística múltiplo $b a-$ ckward descreveu o efeito sob as exposições in- dependentemente, com intervalo de $95 \%$ de confiança (IC95\%). Nesta análise, foram adicionadas as variáveis com valor de $\mathrm{p} \leq 0,20$ na análise univariada, ou que fossem plausíveis teoricamente. Por fim, foram excluídas do modelo múltiplo aquelas colineares (renda, trabalho, número de bens e de pessoas/cômodo). O banco de dados foi construído no programa Epidata 3.1 (Epidata Association, Odense, Dinamarca) e os dados foram analisados no pacote estatístico Stata 10.0 (Stata Corp., College Station, Estados Unidos).

\section{Aspectos éticos}

A pesquisa foi aprovada pelo Comitê de Ética em Pesquisa do Instituto de Saúde Coletiva da Universidade Federal da Bahia (UFBA) (no 045/2010). Todos os entrevistados assinaram o termo de consentimento livre e esclarecido, sendo assegurados o sigilo e a confidencialidade das informações. Foi estabelecido um termo de anuência com as respectivas Secretarias Municipais de Saúde.

\section{Resultados}

Foram coletados dados de 1.290 indivíduos que procuraram algum serviço odontológico no último ano. Destes, 10,16\% não conseguiram atendimento em nenhum nível assistencial, 38,76\% foram ao setor privado, 33,80\% usaram somente a atenção básica e 17,29\%, a atenção básica e CEO. Todos estes últimos responderam que foram encaminhados com uma ficha de referência, entretanto não houve a contra-referência em nenhum dos casos. Dos entrevistados que referiram encontrar barreiras de acesso, 131 procuraram a atenção básica, quatro, o CEO e oito, consultório particular.

A amostra foi composta, predominantemente, por indivíduos do sexo feminino $(62,71 \%)$ não negros (58,91\%), com até 35 anos de idade $(53,88 \%)$ e mais de 7 anos de estudo $(60,16 \%)$, residentes na zona urbana $(71,32 \%)$, não beneficiários de programa social $(62,25 \%)$, que não exercem atividade remunerada $(64,03 \%)$ e com renda familiar maior que um salário mínimo (62,95\%). Em seus domicílios residiam mais de 0,6 pessoas por cômodo $(65,36 \%)$ com até 3 bens $(72,79 \%)$. A maioria estava exposta a um serviço público odontológico melhor organizado $(54,26 \%)$, onde os usuários tiveram maiores dificuldades em su perar as barreiras de acesso $(14,14 \%)$ (Tabela 1$)$.

O perfil de utilização variou conforme o tipo de serviço acessado. A atenção básica foi mais usada pelos entrevistados com mais de 35 anos, em menor posição socioeconômica e residentes 
Tabela 1

Estatística descritiva das variáveis socioeconômicas segundo o tipo de serviço odontológico utilizado, em dois municípios de médio porte da Bahia, Brasil, 2011.

\begin{tabular}{|c|c|c|c|c|c|c|}
\hline Variáveis & $\begin{array}{l}\text { Procurou algum serviço e } \\
\text { não teve acesso (\%) } \\
{[n=131]}\end{array}$ & $\begin{array}{l}\text { Atenção básica (\%) } \\
\qquad[n=436]\end{array}$ & $\begin{array}{l}\text { Serviço privado (\%) } \\
{[\mathrm{n}=500]}\end{array}$ & $\begin{array}{c}\text { Atenção básica e } \\
\text { CEO (\%) } \\
{[n=223]}\end{array}$ & Valor de p & $\begin{array}{c}\text { Total (\%) } \\
{[n=1.290]}\end{array}$ \\
\hline Programa social & & & & & 0,00 & \\
\hline Não & 8,22 & 25,90 & 49,81 & 16,06 & & 62,25 \\
\hline $\operatorname{Sim}$ & 13,35 & 40,04 & 20,53 & 26,08 & & 37,75 \\
\hline Renda (salários mínimos) & & & & & 0,00 & \\
\hline Até 1 & 11,72 & 37,87 & 23,85 & 26,57 & & 37,05 \\
\hline Mais de 1 & 9,24 & 27,34 & 47,54 & 15,89 & & 62,95 \\
\hline Sexo & & & & & 0,00 & \\
\hline Masculino & 11,64 & 27,03 & 46,15 & 15,18 & & 37,29 \\
\hline Feminino & 9,27 & 33,75 & 34,36 & 22,62 & & 62,71 \\
\hline Trabalho & & & & & 0,00 & \\
\hline Remunerado & 10,34 & 24,78 & 50,00 & 14,87 & & 35,97 \\
\hline $\begin{array}{l}\text { Não remunerado ou } \\
\text { desempregado }\end{array}$ & 10,05 & 34,87 & 32,45 & 22,64 & & 64,03 \\
\hline Cor da pele & & & & & 0,56 & \\
\hline Não negra & 10,79 & 30,26 & 38,29 & 20,66 & & 58,91 \\
\hline Negra & 9,25 & 32,64 & 39,43 & 18,68 & & 41,09 \\
\hline Idade (anos) & & & & & 0,00 & \\
\hline Até 35 & 9,21 & 26,04 & 43,60 & 21,15 & & 53,88 \\
\hline Mais de 35 & 11,26 & 37,31 & 33,11 & 18,32 & & 46,12 \\
\hline Escolaridade (anos) & & & & & 0,00 & \\
\hline Até 7 & 12,26 & 40,47 & 26,26 & 21,01 & & 39,84 \\
\hline Mais de 7 & 8,76 & 25,13 & 47,04 & 19,07 & & 60,16 \\
\hline Bens & & & & & 0,00 & \\
\hline Até 3 & 12,03 & 34,61 & 30,56 & 22,79 & & 72,79 \\
\hline Mais de 3 & 5,13 & 22,22 & 60,68 & 11,97 & & 27,21 \\
\hline Número de pessoas por cômodo & & & & & 0,00 & \\
\hline Até 0,6 & 13,20 & 37,81 & 27,07 & 21,92 & & 34,65 \\
\hline Mais de 0,6 & 8,54 & 31,67 & 44,96 & 14,83 & & 65,35 \\
\hline Zona & & & & & 0,03 & \\
\hline Rural & 9,46 & 31,62 & 34,32 & 24,59 & & 28,68 \\
\hline Urbano & 10,43 & 31,09 & 40,54 & 17,93 & & 71,32 \\
\hline Organização do serviço & & & & & 0,00 & \\
\hline Pior & 5,42 & 32,20 & 51,02 & 11,36 & & 45,74 \\
\hline Melhor & 14,14 & 35,14 & 28,43 & 22,29 & & 54,26 \\
\hline
\end{tabular}

CEO: Centro de Especialidades Odontológicas.

no município com serviço de saúde melhor organizado, com uma diferença estatisticamente significante em relação àqueles com o perfil oposto que acessaram os outros níveis assistenciais.

A análise univariada mostrou que entre aqueles que procuraram algum serviço e não conseguiram, ser do sexo feminino $(\mathrm{OR}=0,58$; $\mathrm{IC} 95 \%$ : $0,37-0,90$ ) foi associado à superação das barreiras de acesso, em comparação aos que transitaram pela rede de cuidados à saúde bucal, atenção básica e CEO.
Usar apenas a atenção básica foi associado positivamente aos que têm mais de 35 anos de idade (OR = 1,63; IC95\%: 1,18-2,26), até 7 anos de estudo (OR = 1,64; IC95\%: 1,18-2,27) e residentes no município com um serviço pior organizado $(\mathrm{OR}=1,80$; IC95\%: 1,28-2,53). Por outro lado, ter até 3 bens (OR = 0,63; IC95\%: 0,40-0,98) foi associado a um menor uso da atenção básica, em comparação àqueles que percorreram também a atenção especializada. 
Com relação ao serviço privado, os indivíduos submetidos a uma pior organização do serviço (OR = 3,52; IC95\%: 2,51-4,94) têm três vezes e meia maior utilização, quando comparados aos que usaram o setor público. Associação também positiva foi encontrada entre os residentes em zona urbana (OR = 1,61; IC95\%: 1,15-2,26). Ser beneficiário de programa social $(\mathrm{OR}=0,24$; IC95\%: 0,17-0,36), com renda familiar de até um salário mínimo (OR =0,31; IC95\%: 0,22-0,43), sem atividade remunerada $(\mathrm{OR}=0,44$; IC95\%: $0,32-0,63)$, do sexo feminino ( $\mathrm{OR}=0,54$; IC95\%: $0,38-0,75)$, com menor grau de escolaridade $(\mathrm{OR}=0,54 ;$ IC95\%: 0,40-0,78), número de bens $(\mathrm{OR}=0,22$; IC95\%: 0,14-0,33) e menos pessoas por cômodo no domicílio (OR $=0,41$; IC95\%: 0,29-0,57) foram associados ao menor uso dos consultórios particulares em comparação aos que passaram pelos serviços públicos odontológicos, atenção básica e CEO (Tabela 2).

A análise do modelo final de regressão logística politômica multivariada mostrou que o sexo feminino (OR = 0,58; IC95\%: 0,37-0,92) superou as barreiras de acesso em maior proporção. Ter até 7 anos de estudo (OR = 1,47; IC95\%: 1,03-2,10) e estar exposto a um serviço de saúde menos organizado (OR = 1,74; IC95\%: 1,22-2,48) aumentaram a utilização exclusiva da atenção básica em $47 \%$ e $74 \%$, respectivamente, em comparação àqueles que usaram a atenção básica e o CEO. Para o setor privado, ser do sexo feminino (OR = 0,48; IC95\%: 0,33-0,68), receber programa social (OR = 0,36; IC95\%: 0,25-0,51) e ter menor nível de escolaridade (OR $=0,61$; IC95\%: 0,42-0,88) foram fatores independentemente associados à diminuição do uso deste serviço. Por outro lado, residir em zona urbana $(\mathrm{OR}=1,57$; IC95\%: 1,09-2,26) e estar exposto a uma pior organização do serviço (OR = 3,17; IC95\%: 2,22-4,53) aumentaram este uso, quando comparado aos que percorreram os serviços públicos odontológicos avaliados (Tabela 3). Neste caso, é possível afirmar que a organização do serviço foi o fator mais importante para o uso dos consultórios privados.

Tabela 2

Associação univariada entre variáveis socioeconômicas e a utilização dos serviços odontológicos, tendo como categoria de referência a atenção básica e Centro de Especialidades Odontológicas (CEO), em dois municípios de médio porte da Bahia, Brasil, 2011 ( $n=1.290$ ).

\begin{tabular}{|c|c|c|c|c|c|c|c|c|c|}
\hline \multirow[t]{2}{*}{ Variáveis } & \multicolumn{3}{|c|}{$\begin{array}{l}\text { Procurou algum serviço e não teve } \\
\text { acesso vs. atenção básica e CEO }\end{array}$} & \multicolumn{3}{|c|}{$\begin{array}{c}\text { Atenção básica vs. atenção } \\
\text { básica e CEO }\end{array}$} & \multicolumn{3}{|c|}{$\begin{array}{c}\text { Serviço privado vs. atenção } \\
\text { básica e CEO }\end{array}$} \\
\hline & OR & IC95\% & Valor de $\mathrm{p}$ & OR & IC95\% & Valor de $p$ & OR & IC95\% & Valor de $p$ \\
\hline \multicolumn{10}{|l|}{ Programa social } \\
\hline Sim & 0,94 & $0,61-1,45$ & 0,75 & 0,87 & $0,63-1,20$ & 0,40 & 0,24 & $0,17-0,36$ & 0,00 \\
\hline \multicolumn{10}{|l|}{ Renda (salários mínimos) } \\
\hline Até 1 & 0,78 & $0,51-1,21$ & 0,26 & 0,88 & $0,64-1,21$ & 0,43 & 0,31 & $0,22-0,43$ & 0,00 \\
\hline \multicolumn{10}{|l|}{ Sexo } \\
\hline Feminino & 0,58 & $0,37-0,90$ & 0,01 & 0,95 & $0,67-1,35$ & 0,76 & 0,54 & $0,38-0,75$ & 0,00 \\
\hline \multicolumn{10}{|l|}{ Trabalho } \\
\hline $\begin{array}{l}\text { Não remunerado ou } \\
\text { desempregado }\end{array}$ & 0,67 & $0,42-1,06$ & 0,08 & 0,99 & $0,69-1,42$ & 0,96 & 0,44 & $0,32-0,63$ & 0,00 \\
\hline \multicolumn{10}{|l|}{ Cor da pele } \\
\hline Negra & 1,03 & $0,66-1,61$ & 0,90 & 1,33 & $0,95-1,85$ & 0,09 & 1,23 & $0,89-1,71$ & 0,20 \\
\hline \multicolumn{10}{|l|}{ Idade (anos) } \\
\hline Mais de 35 & 1,44 & $0,93-2,22$ & 0,10 & 1,63 & $1,18-2,26$ & 0,00 & 0,89 & $0,65-1,23$ & 0,48 \\
\hline \multicolumn{10}{|l|}{ Escolaridade (anos) } \\
\hline Até 7 & 1,39 & $0,90-2,16$ & 0,13 & 1,64 & $1,18-2,27$ & 0,00 & 0,56 & $0,40-0,78$ & 0,00 \\
\hline \multicolumn{10}{|l|}{ Bens } \\
\hline Até 3 & 1,01 & $0,54-1,89$ & 0,96 & 0,63 & $0,40-0,98$ & 0,04 & 0,22 & $0,14-0,33$ & 0,00 \\
\hline \multicolumn{10}{|c|}{ Número de pessoas por cômodo } \\
\hline Mais de 0,6 & 1,05 & $0,68-1,61$ & 0,84 & 0,81 & $0,58-1,12$ & 0,20 & 0,41 & $0,29-0,57$ & 0,00 \\
\hline \multicolumn{10}{|l|}{ Zona } \\
\hline Urbano & 1,50 & $0,94-2,42$ & 0,09 & 1,31 & $0,93-1,84$ & 0,12 & 1,61 & $1,15-2,26$ & 0,00 \\
\hline \multicolumn{10}{|l|}{ Organização do serviço } \\
\hline Pior & 0,75 & $0,46-1,23$ & 0,25 & 1,80 & $1,28-2,53$ & 0,00 & 3,52 & $2,51-4,94$ & 0,00 \\
\hline
\end{tabular}

IC95\%: intervalo de 95\% de confiança; OR: odds ratio. 
Tabela 3

Análise de regressão logística múltipla da associação entre variáveis socioeconômicas e a utilização dos serviços odontológicos, tendo como categoria de referência a atenção básica e Centro de Especialidades Odontológicas (CEO), em dois municípios de médio porte da Bahia, Brasil, 2011 ( $=1.290$ ).

\begin{tabular}{|c|c|c|c|c|c|c|c|c|c|}
\hline \multirow[t]{2}{*}{ Variáveis } & \multicolumn{3}{|c|}{$\begin{array}{l}\text { Procurou algum serviço e não teve } \\
\text { acesso vs. atenção básica e CEO }\end{array}$} & \multicolumn{3}{|c|}{$\begin{array}{c}\text { Atenção básica vs. atenção } \\
\text { básica e CEO }\end{array}$} & \multicolumn{3}{|c|}{$\begin{array}{c}\text { Serviço privado vs. atenção } \\
\text { básica e CEO }\end{array}$} \\
\hline & OR & IC95\% & Valor de $p$ & OR & IC95\% & Valor de $p$ & OR & IC95\% & Valor de $p$ \\
\hline Sexo (feminino) & 0,58 & $0,37-0,92$ & 0,02 & 0,96 & $0,67-1,37$ & 0,82 & 0,50 & $0,35-0,71$ & 0,00 \\
\hline Idade (mais de 35 anos) & 1,37 & $0,86-2,19$ & 0,18 & 1,36 & $0,96-1,93$ & 0,08 & 0,85 & $0,59-1,22$ & 0,37 \\
\hline Programa social (sim) & 0,95 & $0,61-1,49$ & 0,83 & 0,95 & $0,68-1,33$ & 0,76 & 0,30 & $0,21-0,44$ & 0,00 \\
\hline Escolaridade (até 7 anos) & 1,26 & $0,79-2,01$ & 0,33 & 1,45 & $1,02-2,07$ & 0,04 & 0,58 & $0,40-0,85$ & 0,00 \\
\hline Cor da pele (preta) & 1,02 & $0,65-1,60$ & 0,93 & 1,28 & $0,91-1,80$ & 0,14 & 1,38 & $0,98-1,96$ & 0,07 \\
\hline Zona (urbana) & 1,48 & $0,91-2,39$ & 0,11 & 1,40 & $0,99-2,00$ & 0,06 & 1,53 & $1,06-2,20$ & 0,02 \\
\hline Organização do serviço (pior) & 0,75 & $0,45-1,23$ & 0,25 & 1,72 & $1,21-2,45$ & 0,00 & 3,24 & $2,27-4,62$ & 0,00 \\
\hline
\end{tabular}

IC95\%: intervalo de 95\% de confiança; OR: odds ratio.

\section{Discussão}

Este estudo revelou que a desigualdade na utilização de serviços públicos odontológicos, em dois municípios baianos de médio porte com $100 \%$ de cobertura da ESF, está associada à escolaridade e à organização dos serviços públicos odontológicos, registrados tanto nas comparações da utilização entre os níveis assistenciais da saúde bucal no SUS (atenção básica $v s$. atenção básica e CEO), quanto entre os serviços públicos e os privados. Nestes últimos, a gestão local, com melhor governabilidade, projeto e capacidade de governo exerce papel importante, já que a melhor organização de serviços públicos odontológicos foi associada a um menor uso da esfera privada. Isso reforça que estes subsistemas não são complementares, mas concorrentes entre si.

A desigualdade foi registrada mesmo quando comparados grupos mais homogêneos, como os usuários da atenção básica e os que percorreram a rede assistencial (atenção básica e CEO). O maior capital cultural, representado pela escolaridade, foi determinante para um uso mais integral dos serviços públicos, quando necessário. Além disso, indivíduos com mais anos de estudo compreendem e percebem melhor as necessidades de saúde. Logo, têm maior poder para superar as barreiras de acesso 6,7.

Uma possível explicação pode estar ancorada na Sociologia Reflexiva de Pierre Bourdieu 27, que analisou a homologia entre forças sociais, estilos de vida e a disputa dos grupos pelo monopólio dos bens públicos. Esta abordagem teórica tem sido defendida e incorporada para o estudo das desigualdades em saúde e da utilização dos serviços 6,7,28. Assim, classes sociais mais altas têm o controle dos bens materiais e simbólicos raros. Serviços de atenção especializada podem ser considerados bens de acesso raro, em disputa pelos diferentes agentes, já que o acesso a eles é apontado como um entrave à garantia da integralidade da atenção 29 .

Ao contrário do esperado, não houve associação entre o capital econômico (maior renda familiar mensal ou não receber benefício social) e melhor uso dos serviços, provavelmente devido a problemas no registro da renda familiar. Não foram encontrados outros estudos que analisassem a desigualdade no uso dos diversos níveis assistenciais da rede pública de serviços odontológicos.

Ainda neste grupo de comparação, um serviço mais organizado - que teve apoio político e planejamento técnico para a sua implementação, que cumpre as diretrizes propostas pela PNSB e com gestores que têm capacidade de governo e controle da condução do processo de trabalho - esteve associado ao maior uso dos serviços públicos odontológicos, facilitando a utilização de uma assistência mais integral. Esta associação esteve presente em outros estudos 10,11,13,14,15 como hipótese explicativa para as barreiras de acesso organizacionais e para a permanência de modelos de atenção tradicionais.

Os resultados da comparação entre uso de serviços públicos e privados mostraram uma tendência pró-equidade, com maior uso da atenção básica e CEO entre as mulheres, os beneficiários de programa social, aqueles com menor escolaridade e residentes em zona rural. Esta tendência de uso pela população mais vulnerável corrobora outros estudos 1,3,19,30. 
Se por um lado o maior uso dos serviços públicos para a população com maior vulnerabilidade social demonstra uma tendência à equidade, por outro sugere uma ESF ainda focalizada, segundo a perspectiva do SUS para pobres 31 . Segundo Paim 31, o SUS tem reduzido as desigualdades no acesso aos serviços e tornado a atenção menos iníqua, embora ainda muito distante do ideário igualitário.

Não foram encontrados outros estudos que analisaram a variável "programa social" como um determinante para a utilização de serviços públicos de saúde. No Brasil, tem-se observado que o benefício social, como o Bolsa Família, é bastante seletivo para identificar pessoas em estado de pobreza 32 . Esta variável foi adicionada ao modelo empírico pelo maior grau de confiabilidade da informação coletada.

A cor da pele não foi associada ao uso dos serviços. Divergindo deste trabalho, um estudo anterior encontrou que idosos negros têm menores chances de utilização dos serviços odontológicos como reflexo da discriminação racial, mesmo quando ajustado por outras variáveis socioeconômicas e de necessidades de saúde 16 .

Mulheres apresentaram maior proporção de uso do serviço público, quando procurado, enquanto homens utilizaram mais o privado. Essa associação é bastante relatada em outros estudos 1,2,3,19. Além de terem uma maior percepção das necessidades de saúde e papel central de cuidadora da família 33, elas ainda registram menor percentual de ocupação 3,22, o que pode explicar este maior acesso.

O percentual de uso dos serviços odontológicos privados $(38,76 \%)$ foi inferior ao registrado na literatura. Um estudo que investigou as capitais do país encontrou, em 21 delas, percentuais maiores que $50 \% 2$. No Brasil, registrouse $48,30 \%{ }^{3}$ e em Campina Grande (Paraíba), $64,30 \%{ }^{30}$. Por outro lado, a utilização dos serviços públicos no presente estudo $(51,09 \%)$ foi maior que o relatado para o Brasil $(46,30 \%$ a $28,90 \%$ para o grupo de 15 a 67 anos), independente da faixa etária 18 , possivelmente em virtude da cobertura de $100 \%$ da ESF. A Região Nordeste é a que mais registra consultas odontológicas no SUS, o que pode ser explicado pela elevada cobertura da ESF. O sudeste do país tem a menor proporção deste tipo de serviço, em contraposição à maior quantidade de beneficiários de planos de saúde odontológicos 34 .

Esta concorrência entre os subsistemas também pôde ser observada neste estudo através da maior facilidade de uso da assistência odontológica privada para os indivíduos expostos a uma pior organização local dos serviços. Neste estudo, pode-se atribuir grande importância desta vari- ável na utilização dos serviços, dado que a sua medida pontual $(\mathrm{OR}=3,24)$ não está contida no intervalo de confiança das outras variáveis. Estudos anteriores sugeriram esta relação entre uso de serviços públicos e o governo local 10,11,13,14,15.

A interpretação da associação entre a variável organização do serviço e o desfecho deve ser feita com cuidado, já que não é possível estabelecer relação causal pela indeterminação da temporalidade dos eventos. É importante destacar que esta é uma variável, por natureza, ecológica, já que cada categoria se refere a um dos municípios pesquisados, e o seu uso no nível individual pode ser configurado como falácia ecológica. Por outro lado, é inegável que todos os indivíduos que residem em uma localidade estão sujeitos à mesma gestão, o que reduz este viés e valida o seu uso. Somente foi viável aplicá-la neste estudo devido à caracterização prévia feita por Rossi 14 . Porém, são necessários outros estudos de caráter confirmatório que investiguem esta associação.

É possível que haja um viés de memória entre os informantes-chaves, já que o responsável pelo domicílio no momento da visita respondeu por todos os residentes com mais de 15 anos sobre o uso dos serviços odontológicos, no período de um ano. Entretanto, os ACS foram orientados a preferencialmente entrevistar as donas de casa e mães que, em geral, sabiam informar os dados requisitados.

A opção metodológica deste estudo, ao eleger municípios com $100 \%$ de cobertura da ESF e realizar inquérito de base domiciliar, teve o intuito de responder, também, lacunas metodológicas 35 e de conhecimento acerca da atenção especializada pública odontológica no país. Além disso, há um melhor desempenho dos CEO nos municípios em que a atenção básica está bem estruturada 10 .

Outros determinantes não investigados neste estudo podem influenciar o acesso e a desigualdade no uso do CEO, como a forma de marcação e a quantidade de consultas, o tempo de agendamento para a primeira consulta e retorno, e a referência e contra-referência 11. Apesar de as barreiras de acesso não terem sido investigadas de modo mais aprofundado, o menor capital cultural parece dificultar o uso da atenção especializada, prejudicando a longitudinalidade do cuidado.

\section{Conclusão}

Este estudo identificou um perfil de desigualdade na utilização de serviços de saúde bucal mesmo entre os usuários do serviço público em diferentes níveis assistenciais. Aqueles que 
têm menor escolaridade e que estão expostos a uma pior organização do serviço usaram menos o CEO. Quando comparado à assistência suplementar, o serviço público apresentou um perfil de vulnerabilidade, embora a pior organização do serviço não favoreça o uso.

Os resultados reiteram que o enfrentamento das desigualdades no acesso e utilização de serviços públicos de saúde é dependente do modo como o governo local articula o projeto e sua capacidade de reorganizar a atenção odontológica. Para isso, sugere-se investir na gestão, qualificando-a. Porém, também cabe aos profissionais prestadores da assistência um olhar mais atento ao perfil de vulnerabilidade dos usuários.

Foi possível verificar uma tendência próequidade no atendimento público odontológico dos municípios pesquisados, o que corrobora os avanços conquistados pelo SUS no âmbito da oferta de serviços. Esta tendência pode camuflar um processo de universalização seletiva, com focalização dos serviços de atenção primária para grupos mais vulneráveis e de atenção especializada para aqueles que possivelmente têm mais capital para romper as barreiras de acesso, sejam elas organizacionais ou socioculturais.

\section{Resumen}

El objetivo de este estudio fue identificar los factores asociados al uso de los servicios odontológicos (primarios y especializados) públicos y privados. Se realizó una encuesta poblacional en dos ciudades de Bahía, Brasil. Los informantes clave contestaron cuestiones socioeconómicas y de utilización de los servicios odontológicos (resultado). La organización de los servicios odontológicos públicos locales fue clasificada en peor/ mejor. Se realizó regresión simple y múltiple con variable politómica. Del total de 1.290 personas, un 38,76\% utilizaron el servicio privado, un 33,80\% la atención primaria y un 17,29\% atención primaria y el Centro de Especialidades Dentales (CED). Una menor escolaridad (OR = 1,47; IC95\%: 1.03-2.10) y una peor organización de servicio $(O R=1,74$; IC95\%: 1,22-2,48) se asociaron con un menor uso de la red de servicios de atención primaria y CED, en comparación con el uso exclusivo de la atención primaria. La desigualdad en el uso de los servicios dentales se observó incluso cuando se comparan grupos más homogéneos, como usuarios de servicios públicos.

Desigualdades en la Salud; Atención Secundaria de Salud; Accesibilidad a los Servicios de Salud; Atención Odontológica; Gobierno Local

\section{Colaboradores}

F. F. Soares contribuiu com a concepção do estudo, análise dos dados e redação do artigo. S. C. L. Chaves e M. C. T. Cangussu contribuíram com a concepção do estudo, análise dos dados e revisão do artigo.

\section{Agradecimentos}

Agradecemos à FAPESB, agência financiadora deste trabalho. 


\section{Referências}

1. Peres KG, Peres MA, Boing AF, Bertoldi AD, Bastos JL, Barros AJ. Redução das desigualdades sociais na utilização de serviços odontológicos no Brasil entre 1998 e 2008. Rev Saúde Pública 2012; 46: 250-8.

2. Peres MA, Iser BPM, Boing AF, Yokota RTC, Malta DC, Peres KG. Desigualdades no acesso e na utilização de serviços odontológicos no Brasil: análise do Sistema de Vigilância de Fatores de Risco e Proteção para Doenças Crônicas por Inquérito Telefônico (VIGITEL 2009). Cad Saúde Pública 2012; 28:90-100.

3. Pinto RS, Matos DL, Loyola-Filho AI. Características associadas ao uso de serviços odontológicos públicos pela população adulta brasileira. Ciênc Saúde Coletiva 2012; 17:531-44.

4. Baldani NH, Antunes JLF. Inequalities in access and utilization of dental services: a cross-sectional study in an area covered by the Family Health Strategy. Cad Saúde Pública 2011; 27 Suppl 2: S272-83.

5. Muirhead VE, Quiñonez C, Figueiredo R, Locker D. Predictors of dental care utilization among working poor Canadians. Community Dent Oral Epidemiol 2009; 37:199-208.

6. Vieira-da-Silva LM. Saúde e espaço social. In: Nogueira RP, organizador. Determinação social da saúde e reforma sanitária. Rio de Janeiro: Centro Brasileiro de Estudos de Saúde; 2010. p. 180-200. (Coleção Pensar em Saúde).

7. Chaves SC, Vieira-da-Silva LM. Inequalities in oral health practices and social space: an exploratory qualitative study. Health Policy 2008; 86:119-28.

8. Coordenação Nacional de Saúde Bucal, Secretaria de Atenção à Saúde, Ministério da Saúde. Diretrizes da Política Nacional de Saúde Bucal. http:// portal.saude.gov.br/portal/arquivos/pdf/politica nacional_brasil_sorridente.pdf (acessado em 01/ Abr/2013).

9. Diretoria de Atenção Básica, Secretaria de Atenção à Saúde, Ministério da Saúde. Brasil Sorridente. Perguntas frequentes. http://dab.saude. gov.br/portaldab/ape_brasil_sorridente.php? conteudo=faq (acessado em 22/Ago/2014).

10. Chaves SCL, Cruz DN, Barros SG, Figueiredo AL. Avaliação da oferta e utilização de especialidades odontológicas em serviços públicos de atenção secundária na Bahia, Brasil. Cad Saúde Pública 2011; 27:143-54.

11. Chaves SCL, Barros SG, Cruz DN, Figueiredo ACL, Moura BLA, Cangussu MCT. Política Nacional de Saúde Bucal: fatores associados à integralidade do cuidado. Rev Saúde Pública 2010; 44:1005-13.

12. Andersen R, Newman JF. Societal and individual determinants of medical care utilization in United States. Milbank Mem Fund Q Health Soc 1973; 51:95-124.
13. Chaves SCL, Soares FF, Rossi TRA, Cangussu MCT, Figueiredo ACL, Cruz DN, et al. Características do acesso e utilização de serviços odontológicos em municípios de médio porte. Ciênc Saúde Coletiva 2012; 17:3115-24.

14. Rossi TRA. A política de saúde bucal na atenção especializada em dois municípios da Bahia: da entrada na agenda à sua implementação [Dissertação de Mestrado]. Salvador: Instituto de Saúde Coletiva, Universidade Federal da Bahia; 2011.

15. Pereira CRS, Patrício AAR, Araújo FAC, Lucena EES, Lima KC, Roncalli AG. Impacto da Estratégia Saúde da Família com equipe de saúde bucal sobre a utilização de serviços odontológicos. Cad Saúde Pública 2009; 25:985-96.

16. Souza EHA, Oliveira PAP, Paegle AC, Goes PSA. Raça e o uso dos serviços de saúde bucal por idosos. Ciênc Saúde Coletiva 2012; 17:2063-70.

17. Araújo SC, Lima RC, Peres MA, Barros AJ. Utilização de serviços odontológicos e fatores associados: um estudo de base populacional no Sul do Brasil. Cad Saúde Pública 2009; 25:1063-72.

18. Secretaria de Atenção à Saúde/Secretaria de Vigilância em Saúde, Ministério da Saúde. SB Brasil 2010. Pesquisa Nacional de Saúde Bucal: resultados principais. http://bvsms.saude.gov.br/bvs/ publicacoes/pesquisa_nacional_saude_bucal.pdf (acessado em 26/Dez/2013)

19. Baldani MH, Almeida ES, Antunes JL. Eqüidade e provisão de serviços públicos odontológicos no estado do Paraná. Rev Saúde Pública 2009; 43: 446-54.

20. Antunes JLF, Narvai PC. Políticas de saúde bucal no Brasil e seu impacto sobre as desigualdades em saúde. Rev Saúde Pública 2010; 44:360-5.

21. Paim J, Travassos C, Almeida C, Bahia L, Macinko J. The Brazilian health system: history, advances, and challenges. Lancet 2011; 377:1778-97.

22. Instituto Brasileiro de Geografia e Estatística. Censo brasileiro 2010. Geociências. http://www.ibge. gov.br/home/download/geociencias.shtm (acessado em 10/Abr/2011).

23. Matus C. Política, planejamento e governo. 2a Ed. Brasília: Instituto de Pesquisa Econômica Aplicada; 1996.

24. Instituto Brasileiro de Geografia e Estatística. Censo brasileiro 2000. Geociências. http://www.ibge. gov.br/home/download/geociencias.shtm (acessado em 10/Abr/2011).

25. Giovanella L, Lobato LVC, Carvalho AI, Conill EM, Cunha EM. Sistemas municipais de saúde e a diretriz para integralidade: critérios para avaliação. Saude Debate 2002; 26:37-61.

26. Kleinbaum DG, Klein M. Logistic regression. A self-learning text. New York: Springer; 2010.

27. Bourdieu P. Razões práticas: sobre a teoria da ação. 8a Ed. Campinas: Papirus; 2007. 
28. Abel T. Cultural capital and social inequality in health. J Epidemiol Community Health 2008; 62:e13.

29. Spedo SM, Pinto NRS, Tanaka OY. O difícil acesso a serviços de média complexidade do SUS: o caso da cidade de São Paulo, Brasil. Physis (Rio J.) 2010; 20:953-72.

30. Rocha RACP, Goes PSA. Comparação do acesso aos serviços de saúde bucal em áreas cobertas e não cobertas pela Estratégia Saúde da Família em Campina Grande, Paraíba, Brasil. Cad Saúde Pública 2008; 24:2871-80.

31. Paim JS. Eqüidade e reforma em sistemas de serviços de saúde: o caso do SUS. Saúde Soc 2006; 15:34-46.

32. Rasella D, Aquino R, Santos CAT, Paes-Sousa R, Barreto ML. Effect of a conditional cash transfer programme on childhood mortality: a nationwide analysis of Brazilian municipalities. Lancet 2013; 382:57-64.
33. O'Donnell O. Access to health care in developing countries: breaking down demand side barriers. Cad Saúde Pública 2007; 23:2820-34.

34. Silva ZP, Ribeiro MCSA, Barata RB, Almeida MF. Perfil sociodemográfico e padrão de utilização dos serviços de saúde do Sistema Único de Saúde (SUS), 2003-2008. Ciênc Saúde Coletiva 2011; 6:3807-16

35. Figueiredo N, Goes PSA. Construção da atenção secundária em saúde bucal: um estudo sobre os Centros de Especialidades Odontológicas em Pernambuco, Brasil. Cad Saúde Pública 2009; 25:259-67.

Submetido em 18/Mai/2014

Versão final reapresentada em 27/Ago/2014 Aprovado em 01/Out/2014 